\title{
CyberCrime Kongress in München
}

\author{
Am 1. und 2. Juli findet im Münchner Marriot Hotel der CyberCrime-Kongress statt. Thema ist der- \\ Umgang mit Cybersicherheit durch proaktives Handeln. Wie können Risiken erkannt und Gefahren \\ bewältigt werden?
}

\section{Cyberkriminialität verändert sich}

Unter anderem geht es darum, dass die Cyberkriminalität sich im Umbruch befindet. Mit der massenhaften Verbreitung von Smartphone, Tablet \& Co. bieten sich den Angreifern neue Ziele und Zugriffswege auf Firmen-IT. Hinzu kommt, dass Angriffe zunehmend von gut organisierten Gruppen durchgeführt werden statt von Einzelnen. Die Software- und Antivirus-Industrie hat bislang keine überzeugende Antwort auf diese Bedrohungen.

Was sind die aktuellen Angriffe die auf Smartphones durchgeführt werden? Wer sind eigentlich die Täter? Sind Passwortsperren und PINs sicher und warum nicht?

Ein weiteres Thema auf dem Kongress ist Wirtschaftsspionage, wer sind die Akteure und wie gehen Sie vor. Und noch wichtiger welche Präventivmaßnahmen lassen sich ergreifen. Trügerisch ist das Argument, in der eigenen Firma gäbe es ja ohnedies nichts zu holen. Eine Schlüsselrolle bei der IT-Sicherheit in Firmen spielt die TK-Anlage. Erfahren Sie was die sicherheitsrelevanten Aspekte bei TK-Anlagen sind und wie diese Anlagen bei der Sicherung der Unternehmens-IT berücksichtigt werden müssen.

\section{Die Inhalte in Stichpunkten:}

Experten-Tipps und Umsetzungshilfen zu den Themen:
- Cybersicherheit durch proaktives Handeln

- Wirtschaftsspionage - bin ich auch betroffen?

- Cyber Forensics - Identifikation von Tätern

- Abwehr von getarnten Angriffen

- Sicherheitsrisiko TK-Anlagen

- Mobile Security - Tatort Smartphone

- Identifizierung der richtigen Penetrationstester

- Schutz von Gebäudeinfrastruktur

- Rechtliche Dimensionen eines Angriffes

\section{Plus interaktive Workshops zu den Themen:}

- Worauf bei Penetrationstest zu achten ist - Ein Leitfaden zur Entscheidungsfindung

- Abwehr von getarnten Angriffen

- IT-Krisenmanagement: Durch Prävention und Reaktion in der Krise erfolgreich bleiben

\section{Fachausstellung "Cyber World 2013}

Parallel zum Fachkongress stellen Anbieter aus dem IT-Sicherheitsumfeld Ihre Produkte und Dienstleistungen vor.

Weitere Informationen zum Kongress unter: http://www.managementforum.com/Veranstaltungen/ CyberCrime/index.php 